\title{
Apparent nuclear transmutations without neutron emission triggered by pseudoprotons
}

\author{
Ruggero Maria Santilli \\ Thunder Fusion Corporation, Tarpon Springs, U.S.A
}

Email address:

research@thunder-energies.com

To cite this article:

Ruggero Maria Santilli. Apparent Nuclear Transmutations without Neutron Emission Triggered by Pseudoprotons. American Journal of Modern Physics. Vol. 4, No. 1, 2015, pp. 15-18. doi: 10.11648/j.ajmp.20150401.13

\begin{abstract}
In this paper, we review the work by Don Carlo Borghi and R. M. Santilli on the laboratory synthesis of the neutron from the proton and the electron under the laws of hadronic mechanics. We then review the prediction of a subsequent synthesis, this time, of the neutron and the electron into a new particle, known as Santilli pseudoproton, which has essentially the same characteristics of the neutron except being negatively charged. We then outline, apparently for the first time, ongoing research on esoenergetic nuclear transmutations without neutron emission that are triggered by a flux of Santilli pseudoprotons.
\end{abstract}

Keywords: Neutrons, Pseudoproton, Antiproton

\section{Introduction}

The synthesis of the neutron from the Hydrogen atom inside stars was proposed in 1920 by $H$. Rutherford [1], experimentally established in 1932 by J. Chadwick [2], and formulated by E. Fermi [3] via his theory of weak interactions according to the familiar form

$$
p^{+}+e^{-} \rightarrow n+v
$$

Systematic theoretical studies on the above synthesis were conducted by R. M. Santilli at the nonrelativistic and relativistic levels [4-7]. The major difficulty originated from the fact that, from the known data

$$
\begin{gathered}
E_{p}=938.272 \mathrm{MeV}, E_{e}=0.511 \mathrm{MeV}, E_{n}=939.565 \mathrm{MeV} \\
E_{n}-\left(E_{p}+E_{e}\right)=0.782 \mathrm{MeV}>0
\end{gathered}
$$

the rest energy of the neutron is $0.782 \mathrm{MeV}$ bigger than the sum of the rest energies of the proton and the electron, thus requiring a "positive binding energy" and resulting in a "mass excess" that are anathema for quantum mechanics, since the sole consistent bound states predicted by quantum mechanics are those characterized by "negative binding energies" resulting in the well known "mass defect".

Santilli solved the problem, firstly, via the construction of a new mathematics today known as isomathematics, and then its use for the construction of a non-unitary covering of quantum mechanics for the invariant description of extended particles moving within physical media under the name of hadronic mechanics (see Refs. [8] for a comprehensive presentation and literature up to 2008). These studies allowed the exact and time invariant representation of all characteristics of the neutron in synthesis (1) at both non-relativistic [4,5] and relativistic [6,7] levels.

The first laboratory synthesis of the neutron according to reaction (1) was done in the late 1960 s by the Italian priest-physicist Don Carlo Borghi and his associates at the University of Recife in Brazil [8]. In the late 1990s, R. M. Santilli conducted systematic tests [9-14] that confirmed the laboratory synthesis of the neutron from a Hydrogen gas, although via principles and apparatus different than those used by Don Borghi.

An important finding during tests [9-14] was the apparent production under minimal condition of power, pressure, etc., of an intermediate state prior to the full synthesis of the neutron called neutroid with the same characteristics of the neutron, except that its spin is zero according to the reaction expressed in standard nuclear units

$$
p_{\downarrow}^{+}+e_{\uparrow}^{-} \rightarrow \tilde{n}(1,0,0,1.008)
$$

Therefore, Santilli introduced in Ref. [9] apparently for the first time a basically new class of nuclides called nucleoids not existing in the Table of Nuclides that are characterized by the absorption of a nuclide by a conventional nuclide according to the rule

$$
\tilde{n}(1,0,0,1.008)+N(A, Z, J, M) \rightarrow \widetilde{N}(A+1, Z, J, M+1.008)
$$


Since the neutroid is unstable when isolated and predicted to decay according to a yet unknown extremely short mean life when absorbed by a nuclide, nucleoids are predicted to have a very short mean life, and to admit decays into conventional nuclides, betas and gammas (see Ref. [9] for details and numerous examples).

The U. S. publicly traded company Thunder Energies Corporation (TEC) with stock symbol TRNG is currently setting up the production and sale of a Thermal Neutron Source (TEC-TNS-14003) for the production where and when desired of thermal neutrons at the desired flux and energy (see Refs. [13-15] for details).

Keynote lectures on the above aspects are available from Refs. [16]; and independent review specifically devoted to the neutron synthesis is available in Ref. [17]; and a general independent review is available from monograph [18].

Following the mathematical, theoretical and experimental understanding of the synthesis of the neutron from a Hydrogen gas, Santilli proposed in Ref. [19], that Rutherford compression of the electron within the proton can occur a second time, resulting in a new particle, known as Santilli pseudoproton and denoted with the symbol $\bar{p}^{-}$, possessing spin $1 / 2$, negative charge, esentially the same charge radius, rest energy, mass and mean life of the neutron, according to the synthesis

$$
\left(p_{\downarrow}^{+}+e_{\uparrow}^{-}\right)+e_{\downarrow}^{-} \rightarrow \bar{p}^{-}(1,-1,1 / 2,1.008)
$$

where: we should note the appearance, apparently for the first time in nuclear data, of the negative charge of the pseudoproton; we have ignored in first approximation the correction of the neutron mass due to the rest energy of the electron me $=0.0005 \mathrm{amu}$; and one should note the lack of need for the emission of a neutrino in agreement with Refs. [3-6].

It is evident that the absorption of a pseudoproton by a tabulated nuclide creates a nucleoid according to the rule

$$
\bar{p}^{-}(1,-1,1 / 2,1.008)+N(A, Z, J, M) \rightarrow \widetilde{N}(A+1, Z, J, M+1.008)+\beta^{-}
$$

where we have assumed the virtually instantaneous decay of the pseudoproton when absorbed by a nuclide, with consequential beta emission. The existence of the pseudoproton was additionally studied in the paper [13], where it is noted that the experimental set up for the synthesis of the neutron from a Hydrogen gas appears to be suited for the production of Santilli pseudoproton.

This is due to the fact that the neutrons synthesized by the DC arc generally have low (thermal) energy and, therefore, are predicted to remain within the plasma surrounding the DC arc for a time sufficient to allow a second synthesis according to reaction (5). Needless to say, in the event produced, pseudoprotons cannot be detected outside the reactor due to their charge.

Consequently, the resolution as to whether the synthesis of the neutron also synthesizes Santilli's pseudoprotons requires the construction of a sufficiently large, specially designed, reactor containing in its interior all necessary equipment for:

1) The separation of positively and negatively charged particles expectedly via positively and negatively charged plates with sufficiently strong different of potential;

2) The separation of electrons from the pseudoprotons expectedly via their large mass difference and possibly via mass spectrometers; and

3) The elimination from all detections of counts caused by Hydrogen atoms in one of its ionized forms and other conditions.

The plausibility of the existence of Santilli pseudoproton and a detailed study of its detection means has been confirmed by V. de Haan in the recent paper [20].

In this paper, we indicate that, subject to independent confirmations and expected numerous additional developments, the energy output of Hydrogen based nuclear transmutations (see, e.g., representative tech- nologies [21, 22], can be enhanced to such a level to achieve industrial value.

\section{Nuclear Transmutations Triggered by Santilli Pseudoprotons}

Some of the most interesting nuclear energies are those based on Hydro- gen to trigger esoenergetic transmutations of stable nuclides into stable nuclides without the emission of neutronic or other damaging radiations and without the release of radioactive waste.

The biggest difficulty in these studies is that the proton (considered as the nucleus of the Hydrogen atom) is protected in nature by a spherical distribution of the peripheral electron orbits. Following ionization of the Hydrogen atom, there exist evident difficulties to achieve an industrial valuable energy output because the proton is positively charged and, as such, it is repelled by the positive charge of the selected nuclide.

These features essentially imply that the desired nuclear transmutations can at best be at random. II any case, no energy output sufficiently large to have industrial value has been achieved to date via nuclear transmutations stimulated by Hydrogen and none can be expected in the near future to our best knowledge.

An interesting approach to resolve the proton of the Coulomb repulsion between the proton of the Hydrogen atom and the selected nuclide has been recently proposed by U. Abundo [23] according to which the problem of the Coulomb repulsion can be bypassed by the synthesis of the Hydrogen into Santilli nucleoids (3) [9] because, being neutral, they can be readily absorbed by nuclides resulting into unstable Santilli nucleoids as per reaction (4) with conventional esoenergetic decays.

In this paper, we propose apparently for the first time the research and development under way at the U. S. publicly traded company Thunder Energies Corporation on the stimulation of nuclear transmutations via the Santilli 
pseudoprotons (5) [19]. Since the pseudoprotons are negatively charged, they are attracted by the positively charged nuclides. Once physical contact has occurred between the pseudoproton and the nuclide, strongly attractive nuclear forces take over resulting in the absorption of the pseudoproton.

As recalled in Section 1, the mean life of the pseudoproton when isolated is unknown, but it can be safely assumed in first approximation to be that of the neutron (15 minutes). However, when the pseudoproton is absorbed by the nuclide resulting in the formation of an extremely unstable nucleoid, the pseudoproton is expected to decay almost instantly into the proton with the expulsion of the excess electron.

Additionally, the charged character of pseudoprotons merits their fully controlled industrial production in a way definitely preferable to that of the neutroids. We should finally indicate that the controlled production of pseudoprotons via electric discharges can be done via available tech- nologies, such as those developed for the claimed antiprotons.

One illustration among several possible not presented for brevity, is given by the nuclear transmutation of the Li-7 stimulated by a pseudoproton according to the sequential reactions

$$
\begin{gathered}
\bar{p}^{-}(1,-1,1 / 2 \uparrow, 1.008)+\operatorname{Li}\left(7,3,3 / 2_{\downarrow}, 7.016\right) \rightarrow \\
\rightarrow \operatorname{Li}(8,3,3 / 2,8.0225)+\beta^{-} \rightarrow \\
\rightarrow \operatorname{Be}(8,4,2,8.0225)+\beta^{-} \rightarrow \\
2 \mathrm{He}(4,2,1,4.0026) \Delta \mathrm{E} \\
\Delta \mathrm{E}=2.887 \times 10^{-12} \mathrm{~J}
\end{gathered}
$$

where one should note: the antiparallel spin polarizations of the pseudo- proton and the Lithium to trigger the release of the excess electron; the necessary intermediate production of the nucleoid $\tilde{\operatorname{Li}}(8,4,3 / 2,8.0225)$ that does not exist in the standard Table of Nuclides; and the produc- tion of two electrons that can be used to collect additional heat and crate separate source of electric energy.

In this case, we have the energy output (7b) per stimulated nuclear transmutation (where we ignore in first approximation the energy input and the beta emissions) with total energy output per hour predicted in Ref. [9]

$$
\mathrm{E}_{\text {out }}=\left(2.88 \times 10^{-12} \mathrm{~J}\right) \times 10^{16}=2.8 \times 10^{4} \mathrm{~J} / \mathrm{min}=1.7 \times 10^{6} \mathrm{~J} / \mathrm{h}
$$

which, in case confirmed, has evident industrial value because the indicated considerable energy output per hour can be achieved with a rate of e.g., $10^{16}$ stimulated transmutations per hour on $10^{23}$ nuclei in a mole of Li-7, namely, with a efficiency rate of $10^{-7}$.

These conditions are rather reasonable, for instance, for the use of a collimated beam of thermal pseudoprotons hitting a rod of Li-7 along its symmetry axis, the dispersal of the pseudoproton bean on Lithium plate, and other configurations (international patent pending by Thunder Energies Corporation).

Additionally, one should note the truly controlled character of stimulated transmutations (7) in regard to their initiation, energy output, and termination via the corresponding control of the flux of Santilli pseudo- protons.

It may be of some value to indicate the additional nuclear transmutations currently under study of Li-7

$$
\begin{gathered}
\bar{p}^{-}(1,-1,1 / 2 \uparrow, 1.008)+\operatorname{Li}\left(7,3,3 / 2_{\downarrow}, 7.016\right) \rightarrow \\
\rightarrow \operatorname{Li}(8,3,3 / 2,8.0225)+\beta^{-} \rightarrow \\
\rightarrow \operatorname{Be}(8,4,2,8.0225)+\beta^{-} \rightarrow \\
2 \operatorname{He}(4,2,1,4.0026)+\Delta \mathrm{E}
\end{gathered}
$$

of Pd-106

$$
\begin{gathered}
\bar{p}^{-}(1,-1,1 / 2,1.008)+\operatorname{Pd}\left(106,46,0^{+}, 105.903 \rightarrow\right. \\
\rightarrow \widetilde{\operatorname{Pd}}\left(107,46,0^{+}, 105.911+\beta^{-} \rightarrow\right. \\
\rightarrow \operatorname{Ag}\left(107,47,1 / 2^{+}, 106.9050\right)+\beta^{-}+\Delta E
\end{gathered}
$$

and of Au-197

$$
\begin{gathered}
\bar{p}^{-}(1,-1,1 / 2,1.008)+\operatorname{Au}(197,79,3 / 2,196.966) \rightarrow \\
\widetilde{A} u(198,79,3 / 2,196.974)+\beta^{-} \rightarrow \\
\rightarrow \operatorname{Au}(198,79,2,197.972)+\beta^{-}+\Delta E \rightarrow(11)
\end{gathered}
$$

where $\mathrm{Au}-198$ is unstable and decay in 2.69 days into betas and $\mathrm{Hg}-198$ with the release of $1.372 \mathrm{MeV}$.

The industrially relevant aspect is thatb in none of Reactions (7)-(11) there can be any production of harmful neutronsm, although the production of all types of electromagnetic waves, all the way to energetic gammas, have been systematically measured.

To prevent unrealistic expectations of a rapid production of the new clean hadronic energy, we should stress the need to resolve a number of engineering problems, such as the efficient production of the needed pseudoproton flux, the efficient use of the produced heat, the elimination of possible, locally explosive events, and others.

Nevertheless, we can realistically state that the plausibility of the existence of the pseudoprotons is established buy the very existence of the neutron and that, apparently for the first time to our knowledge, more adequate mathematical and physical theories have apparently resolve major objections against the achievement of much needed, industrially valuable, nuclear energies without harmful radiations.

\section{Conclusion}

Following three quarter of a century of attempts in the achievement of low and high energy nuclear syntheses without reaching industrially valuable results despite combined expenditures estimated to be of the order of trillions of dollars, it is time to admit the need for new vistas in the search of new clean nuclear energies.

Above all, there is the need to admit that the lack of achievement of industrially valuable results may be due to 
insufficiencies of quantum mechanics for nuclear structures and reactions, such as the notorious insufficiencies for the synthesis of the neutron, the representation of basic nuclear data, such as nuclear spins and magnetic moments, the reversible character over time of quantum mechanics compared to the strict irre- versibility over time of all nuclear reactions, and other insufficiencies.

We hope that this paper may stimulate young minds of any age to use of new vistas in the search for new clean, industrially valuable nuclear energies, firstly, by using a more adequate mechanics resolving the insuf- ficiencies of quantum mechanics and, secondly, by searching for new optimization means that, by conception, cannot be even predicted by quantum mechanics, but are fully predictable and quantitatively treatable by the covering hadronic mechanics and related isomathematics.

\section{References}

[1] H. Rutherford, Proc. Roy. Soc. A, 97, 374 (1920).

[2] J. Chadwick, Proc. Roy. Soc. A, 136, 692 (1932).

[3] E. Fermi, Nuclear Physics, University of Chicago Press (1945).

[4] R. M. Santilli, “Apparent consistency of Rutherford's hypothesis on the neutron as a compressed Hydrogen atom, Hadronic J.13, $513 \quad$ (1990). http://www.santilli-foundation.org/docs/Santilli-21.pdf

[5] R. M. Santilli, “Apparent consistency of Rutherford's hypothesis on the neutron structure via the hadronic generalization of quan- tum mechanics - I: Nonrelativistic treatment", ICTP communication IC/91/47 (1992). http://www.santilli-foundation.org/docs/Santilli-150.pdf

[6] R. M. Santilli, "Representation of the synthesis of the neutron inside stars from the Hydrogen atom.", Communication of the Joint Institute for Nuclear Research, Dubna, Russia, number JINR-E4-93-352 (1993).

[7] R.M. Santilli, "Recent theoretical and experimental evidence on the apparent synthesis of neutrons from protons and electrons," Chinese J. System Engineering and Electronics 6, 177

http://www.santilli-foundation.org/docs/Santilli-18.pdf

(1995).

[8] C. Borghi, C. Giori C. and A. Dall'Olio, Communications of CENUFPE, Number 8 (1969) and 25 (1971), reprinted via Santilli's proposal in the (Russian) Phys. Atomic Nuclei, $<\mathrm{b}>$ $56</$ b $>$, 205 (1993).

[9] R. M. Santilli, "Apparent confirmation of Don Borghis experiment on the laboratory synthesis of neutrons from protons and electrons", Hadronic J. 30, 29 (2007). http://www.i-b-r.org/NeutronSynthesis.pdf

[10] R. M. Santilli, Confirmation of Don Borghis experiment on the synthesis of neutrons, arXiv publication, August 15, (2006). http://arxiv.org/pdf/physics/0608229v1.pdf
[11] R. M. Santilli, "The synthesis of the neutron according to hadronic mechanics and chemistry," Journal Applied Sciences 5,32 (2006).

[12] R. M. Santilli, "Documentation of scans from the Polimaster and SAM 935 detectors during tests [11-13] http://www.neutronstructure.org/neutron-synthesis-3.htm www.neutronstructure.org/neutron-synthesis-2.htm

[13] R. M. Santilli and A. Nas, "Confirmation of the Laboratory Synthesis of Neutrons from a Hydrogen Gas," Journal of Computational Methods in Sciences and Engineering, in press (2014)

[14] www.Thunderengies.com / docs / neutron - synthesis - 2014 . pdf

[15] R. M. Santilli and A. Nas, 12 minutes film on "Laboratory Synthesis of Neutrons from a Hydrogen Gas", www.world-lecture-series.org/newtron-synthesis-08-14

[16] P. Fleming, "Summary of the novel technologies of Thunder Energies Corporation," website

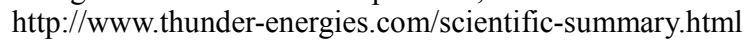

[17] R. M. Santilli, 2014 Keynote Lectures, IsoLinear IsoInvariant isoRelativity, Limitations of Special Relativity and Quantum Mechanics

http://www.world-lecture-series.org/isorelativity-2014-i Experimental Verifications in Particle Physics http://www.world-lecture-series.org/isorelativity-2014-ii Experimental Verifications in Nuclear Physics http://www.world-lecture-series.org/isorelativity-2014-iii

[18] J. V. Kadeisvili, “The Rutherford-Santilli Neutron,” Hadronic J vol. 31, p. 1, 2008; and website http://www.i-b-r.org/Rutherford-Santilli-neutron.htm

[19] I. Gandzha and J Kadeisvili, New Sciences for a New Era: Mathematical, Physical and Chemical Discoveries of Ruggero Maria Santilli, Sankata Printing Press, Nepal (2011), $\mathrm{http} / /$ www.santilli-foundation.org/docs/RMS.pdf

[20] R. M. Santilli, "Apparent detection of antimatter galaxies via a refractive telescope with concave lenses. "Clifford Analysis, Clifford Algebras and their Applications, 3, 1 (2014) http://www.santillifoundation.org/docs/Antimatter-telescope-2013-final.pdf

[21] V. de Haan, "Possibilities for the detection of Santilli's pseudoprotons," Submitted for publication http://www.santillifoundation.org/docs/pseudoproton-2014.pdf

[22] S. Focardi and A. Rossi, "A new energy source from nuclear fusion", Journal of Nuclear Physics, February 28th, 2010, published online at http://www.journal-of-nuclear-physics.com/?m=201002.

[23] R. E. Godes, "The quantum reaction hypothesis," http://www.lenrcanr.org/acrobat/GodesRquantumfus.pdf

[24] Ugo Abundo, "Interpretation and enhancement of the excess energy of Rossi's reactor via Santilli neutroids and nucleoids," Hadronic Journal Vol. 37, pages 697-737 (2014, pdflink 106 MB

http://www.thunder-energies.com/docs/abundo-paper-2014.pd $\mathrm{f}$ 